10,000 females. The syndrome is caused by a mutation of the MECP2 gene, which is located on the X chromosome. Patients have abnormal neuronal morphology, but the neurons do not die. Investigations in mice have now given hope that these defective neurons can be repaired, and symptoms reversed.

Guy et al. silenced the mouse Mecp2 gene through insertion of a lox-Stop DNA cassette. Mecp2-silenced animals were bred with mice in which Cre recombinase was present in the cytoplasm of every cell. After the male offspring had developed the symptoms of Rett syndrome, administration of the estrogen analog tamoxifen caused the Cre recombinase to migrate to the nucleus, where it was able to excise the lox-Stop cassettes, thereby reactivating Mecp2.

Although intensive dosing of tamoxifen caused neurological symptoms followed by death in 9 of 17 mice, more-gradual activation (through weekly injection of tamoxifen followed by booster treatments) prevented this toxic effect. Additionally, over 4 weeks, 5 of 6 symptomatic mice were rescued with tamoxifen therapy, exhibiting decreased severity of symptoms (inertia, gait disturbances, hind-limb clasping, tremor, irregular breathing, increased weight) and increased survival. Symptoms were reversible even in mature mice with wellprogressed symptoms. The treatment also normalized long-term potentiation (critical to behavior and learning), which is defective in mouse models of Rett syndrome.

These experiments suggest that Rett syndrome is not, as previously suspected, a strictly neurodevelopmental disorder. It is possible that the symptoms associated with the disorder could also be reversible in humans-even those with well-developed disease.

Original article Guy J et al. (2007) Reversal of neurological defects in a mouse model of Rett Syndrome. Science 315: $1143-1147$

\section{No link between mobile phone use and increased risk of glioma}

Mobile phones emit low-energy radiofrequency radiation. Although no mechanism for the initiation of cancer via radiofrequency radiation has been established, there has been widespread anxiety about a possible link between mobile phone use and an increased risk of intracranial tumors. In a recent paper, Lahkola et al. report the results of a population-based case-control study, conducted in Denmark, Finland, Norway, Sweden and southeast England, designed to evaluate the relationship between mobile phone use and risk of glioma.

A total of 1,521 patients with glioma and 3,301 controls participated in this study. No significant association was found between risk of glioma and regular mobile phone use. Subgroup analyses found no association between risk of glioma and cumulative number of calls, cumulative call hours, lifetime years of use, or the use of digital or analogue telephones. Similar results were found for men and women and across age groups. Laterality analysis demonstrated an association of borderline significance between a more than 10-year reported use of a mobile phone on one side of the head and tumor location $(P=0.04$ for trend).

The authors conclude that while their results do not indicate an association between mobile phone use and glioma, further studies of the risk associated with long-term mobile phone use should be carried out, with particular attention paid to the incidence of tumors in the areas of the brain most heavily exposed to radiofrequency radiation during mobile phone use.

Original article Lahkola A et al. (2007) Mobile phone use and risk of glioma in 5 North European countries. Int $J$ Cancer 120: 1769-1775

\section{Study finds no evidence to support restriction of seafood consumption during pregnancy}

In the US, pregnant women are advised to restrict their consumption of seafood to no more than $340 \mathrm{~g}$ per week to limit fetal exposure to neurotoxic pollutants; however, seafood is an important source of omega-3 fatty acids, which are thought to be essential for optimum neurological development. Using data from the Avon Longitudinal Study of Parents and Children (ALSPAC), Hibbeln et al. examined the relationship between seafood consumption during pregnancy and neurological development in childhood.

In total, 11,875 pregnant women living in Bristol and surrounding areas in the UK completed a questionnaire assessing seafood 